\title{
Laboreal
}

Volume $12 \mathrm{~N}^{\circ} 1$ | 2016

Os Equipamentos de Proteção Individual (EPI):

protetores, mas nem sempre

\section{Maurice Reuchlin y la dialéctica de laboratorio / campo}

Maurice Reuchlin e a dialética laboratório / terreno

Maurice Reuchlin et la dialectique laboratoire / terrain

Maurice Reuchlin and the dialectics lab / field

Jacques Leplat

Traductor: Cecilia De la Garza y Mario Poy

\section{(2) OpenEdition}

Journals

Edición electrónica

URL: http://journals.openedition.org/laboreal/3370

DOI: 10.4000/laboreal.3370

ISSN: 1646-5237

Editor

Universidade do Porto

Referencia electrónica

Jacques Leplat, « Maurice Reuchlin y la dialéctica de laboratorio / campo », Laboreal [En línea], Volume $12 N^{0} 1$ | 2016, Publicado el 01 julio 2016, consultado el 24 septiembre 2020. URL : http:// journals.openedition.org/laboreal/3370; DOI : https://doi.org/10.4000/laboreal.3370

Este documento fue generado automáticamente el 24 septiembre 2020

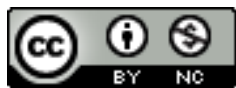

Laboreal está licenciado com uma Licença Creative Commons - Atribuição-NãoComercial 4.0 Internacional. 


\title{
Maurice Reuchlin y la dialéctica de laboratorio / campo
}

\author{
Maurice Reuchlin e a dialética laboratório / terreno \\ Maurice Reuchlin et la dialectique laboratoire / terrain \\ Maurice Reuchlin and the dialectics lab / field
}

Jacques Leplat

Tradución : Cecilia De la Garza y Mario Poy

\section{NOTA DEL EDITOR}

http://dx.doi.org/10.15667/laborealxii0116jles

1 La elección del texto que aquí se presenta responde a un doble objetivo : proponer un tema que mantiene siempre su interés, así como rendir homenaje a un gran psicólogo, Maurice Reuchlin (1920 -2015), fallecido recientemente luego de una larga enfermedad que lo condenó prematuramente al silencio. Este investigador produjo una obra significativa plasmada en libros y artículos. Citemos dos ejemplos: un tratado de Psicología reeditado varias veces y un Tratado de psicología aplicada (trabajo colectivo del cual fue su director) que incluye una decena de volúmenes que cubren los principales campos de la psicología aplicada. No se trata, acá, de hacer un balance de estos trabajos, otros lo realizarán, sino de proponer un texto que se inscriba dentro del período en el cual fue escrito y dentro del campo de interés del autor. Este comentario se centrará en el texto en sí y en el desarrollo de las ideas que defendió. Se hará también alusión a los campos de investigación relacionados con esas ideas, susceptibles de enriquecerlas.

2 El texto elegido fue publicado en un número de Le Travail Humain de 1978 (Volumen 41, fasc.2, pp. 307-324) y forma parte de un ciclo de estudios organizado en el marco de la III Sección de la École Pratique des Hautes Études (EPHE). Los autores de los textos de este ciclo que aparece en el citado número tenían responsabilidades importantes en los 
laboratorios de psicología de la EPHE, agrupados en el mismo edificio, y deseaban mostrar a través de dicho ciclo que, a pesar de la diversidad y de las respectivas orientaciones, compartían preocupaciones comunes. El tema general del dicho ciclo de estudios, "La investigación psicológica en el laboratorio y en el campo" no fue elegido al azar. En efecto, en ese momento, habían tenido lugar numerosos debates, en particular dentro de la Sociedad Francesa de Psicología que, en 1969, organizó su reunión anual en torno a este tema. Para establecer claramente el contexto en el que se inscribe este texto, se ha considerado pertinente mencionar el título y los autores de los trabajos presentados en este ciclo y sus contenidos, figurando ellos también en el citado número de Le Travail Humain.

R. Zazzo.- El estudio del niño en el laboratorio y en situaciones habituales.

H. Gratiot Alphandéry.- ¿La escuela es un campo de estudio?

F. Bacher.- Las encuestas : contribuciones y límites de un método de estudio de campo.

J. Leplat.- La equivalencia de las situaciones de laboratorio y de campo.

7 M. Reuchlin.- Tentativa de análisis de la distinción " Psicología en el laboratorio Psicología en el campo".

Sobre el tema de este ciclo de estudios, se encontrarán resonancias en muchos textos de Reuchlin mencionados en la siguiente sección. Hay uno, particularmente significativo, titulado "¿La psicología aplicada posee métodos propios? "En el capítulo introductorio del tomo 2 del Tratado de Psicología Aplicada, del cual el autor tuvo a cargo la dirección. También es de remarcar que Reuchlin, en el texto al cual se alude acá, transformó un poco el título dado en la presentación de los trabajos publicados, "La investigación psicológica en el laboratorio y en el campo", resultando en "...psicología en el laboratorio, psicología en el campo" . No vamos a discutir esta "traducción", pero nos gustaría destacar la elección que hicimos para centrar el foco de nuestros comentarios sobre el concepto de la psicología aplicada que engloba a la anterior y permite situarla mejor.

9 Una parte importante de la obra de Reuchlin fue dedicada a la psicología aplicada, en particular, en el Tratado en el cual redactó varios capítulos y que, lamentablemente, fueron olvidados con demasiada rapidez. Los 10 tomos del tratado cubren un amplio campo, tal como lo demuestra su título: Aplicaciones de la psicología. Los métodos de la psicología aplicada. Trabajadores y sistemas técnicos. Trabajadores y empleos. La educación y el desarrollo individual. Las instituciones educativas. El diagnóstico de las discapacidades y la rehabilitación. Aplicaciones médicas. La psicología social. La vida diaria. Cualquier trabajo que se considere serio en una de estas áreas no puede ignorar estos textos de los cuales, cada uno, ha sido objeto de un riguroso examen crítico. Yo mismo, habiendo escrito varios capítulos, puedo dar testimonio de este Tratado habiendo podido apreciar la calidad y la transparencia con la que se condujeron las discusiones previas a la producción de los textos.

El prólogo al primer tomo sobre "las aplicaciones de la psicología" esboza algunos problemas planteados por la concepción de una obra tal y justifica las opciones elegidas y se encuentran resonancias de temas e ideas discutidos en el texto de referencia aquí comentado. En primer lugar, la afirmación con la que abre el primer párrafo: "la psicología aplicada tomando a la persona en su contexto de vida cotidiano, es directamente dependiente de la evolución general de la sociedad". Luego, esta declaración a la cual volveremos, "el tema más central es el estatuto mismo de la 
psicología aplicada. Algunos tienden a considerarla como la ciencia del hombre en su entorno de vida habitual; otros como la aplicación de una ciencia psicológica fundamental, teórica, pura, experimental ; otros, finalmente la consideran una práctica concreta haciendo irrisoria cualquier referencia a la ciencia "(p. 6). Es posible, también, distinguir "una tendencia a prestar atención a las diferencias individuales y la tendencia orientada hacia el estudio de las condiciones del contexto impuesto a todos los individuos de un grupo (condiciones de trabajo, los métodos de enseñanza, etc.) "(p. 7).

11 El segundo capítulo del primer tomo se titula "El nacimiento de la psicología aplicada" y constituye un tipo de historia de la psicología aplicada vista a través de las corrientes teóricas en las cuales se han desarrollado las investigaciones, en este período: la psicología funcionalista, la psicología estructuralista, la psicología dinámica. Para estar más cerca de la temática de este comentario, se ha considerado conveniente retomar el concepto de la psicología aplicada cuyas ambigüedades son la fuente de los problemas que revelan la dificultad de identificar el lugar a otorgarle a la psicología, en el estudio de las situaciones en la cuales se desarrollan las actividades humanas. A continuación, se trataran dos nociones que pueden enriquecer el debate, propio al texto comentado.

\section{El concepto de la psicología aplicada}

Las investigaciones psicológicas sobre las situaciones de trabajo $\mathrm{y}$, de manera más general, sobre "las condiciones habituales de vida ", para retomar la expresión de Reuchlin, se clasificaron inicialmente bajo el nombre de psicotécnica. Lahy en Francia fue el gran precursor y, bajo esta etiqueta, produjo muchos trabajos. Encontraremos referencias en los textos sobre la historia de la psicología del trabajo, sobre todo en un número especial de la revista "Psychologie française" consagrado al centenario de la Sociedad Francesa de Psicología, y también en textos que Suzanne Pacaud publicó, sola $\mathrm{y}$ en colaboración con Lahy, numerosos textos bajo la etiqueta de la psicotécnica $y$, posteriormente, de psicología aplicada. La palabra psicotécnica figura también en el nombre de las instituciones en las cuáles trabajaban los psicólogos investigadores. Es así que, Faverge (1912-1988) en coautoría con Ombredane (1898-1958) del libro sobre el análisis del trabajo (1955), trabajó en una organismo llamado "Centre d'Études et de Recherches Psychotechniques" (CERP), que pertenecía a una asociación profesional denominada " Association professionnelle des psychotechniciens diplômés". Pero en el momento en que se desarrollaba la formación de los psicólogos laborales, el término psicotécnico tenía mala prensa debido a la palabra técnico, considerada desvalorizante por las partes interesadas. Fue entonces que apareció y se generalizó la denominación de psicología aplicada. Pero esto, a su vez, se reveló poco satisfactorio. Lahy mismo (1932), señaló que "ahora que la psicología llamada aplicada ha superado el período de las primeras aplicaciones, podemos plantear -en toda su extensión- el problema de sus fundamentos" (Lahy (1932, p. 11). Entre estos están: "¿Es la llamada psicología aplicada solamente "aplicada ", o debe ser considerada como la psicología general tanto teórica como aplicada ?" (id.).

Wallon (1930/1946), por su parte, denuncia las ambigüedades y malas interpretaciones de la denominación de la psicología aplicada. "La psicología aplicada no consiste en desplazar al dominio de las aplicaciones prácticas los principios o verdades de la psicología que se dice teórica, racional o que, simplemente, se funda en la introspección" (Wallon, 1930/1946, 
p. 7). De hecho, la palabra aplicación lleva consigo la idea de que existe un conjunto de conocimientos directamente utilizables para el diseño de una intervención. Ahora bien, esta idea es cuestionable. Si bien es cierto que la intervención hace uso de conocimientos previos, es necesario primero determinar qué conocimientos se necesitan utilizar : antes de la aplicación, por lo tanto, existe una fase de análisis que requiere en sí de conocimientos previos. Las investigaciones ergonómicas son las que, justamente, iban a poner en evidencia la importancia de este paso preliminar que será designado como la etapa de diagnóstico, la etapa previa a la implementación o al desarrollo de los conocimientos que guiará la intervención. El libro de Guerin et al. (1997) ha presentado con particular esmero y sumamente ejemplificado, el lugar del diagnóstico en la intervención ergonómica.

Es importante tener en cuenta una posible deriva en el uso del concepto de aplicación, el cual era común en el mundo académico en el momento del desarrollo de la psicología ergonómica y del cual, tal vez, se encuentren rastros actualmente. Esta deriva se caracteriza por la opinión de que cualquier investigación de campo, es decir, en las condiciones habituales de la vida y del trabajo, es una investigación aplicada : hubo un momento en que esta opinión era común y difícil de combatir.

En ese período, paralelo al del desarrollo de la psicología cognitiva, (1982), habíamos publicado un artículo titulado "El estudio de campo, desafío (u obstáculo) al desarrollo de la psicología cognitiva." Este texto llevó a definir con precisión las características de los estudios de campo en comparación con los estudios de laboratorio, al mismo tiempo que mostrar la necesidad de articular las investigaciones llevadas a cabo en ambos dominios. En la conclusión se señaló : "Por lo tanto, no se hablará de psicología de campo, ni de psicología de laboratorio, como si se tratara de dos psicologías diferentes. Los estudios de campo y el laboratorio son sólo dos lugares donde debería desarrollarse, de manera coordinada, la psicología. "(P.127). Otra ventaja de este texto es que fue objeto de observaciones, de críticas detalladas de los investigadores franceses y extranjeros a los que se les pidió comentarios e informes que enriquecieran el debate sobre los dos términos, campo / laboratorio.

La cuestión debatida por ese entonces continuó los años siguientes, en particular en el campo de la didáctica profesional. Existe, en particular, un libro muy claro y bien documentado de Pastré (2011), "La didactique professionnelle" (subtítulos: enfoque antropológico de la formación de adultos. Formación y práctica profesional), el cual retomaremos.

\section{El rol del contexto}

17 El análisis de campo de las situaciones de trabajo reveló el importante papel desempeñado por el contexto. Leplat $(2002,2006)$ ha dedicado a este tema dos revisiones que ofrecen una extensa bibliografía que debería ser actualizada, pero que ya proporciona los elementos esenciales para el diseño de una investigación sobre el tema o, más simplemente, para tomar en cuenta el papel de esta característica relacionada con cualquier situación de trabajo o práctica. Hollnagel (1993) desarrolló un modelo de control de la acción a través del contexto, a partir de la idea de que "las acciones sólo tienen sentido en un contexto" (p. 164) y que éste puede guiar la ejecución de la acción. La noción de contexto está estrechamente vinculada al problema de la posibilidad de generalización de los resultados de un estudio llevado a cabo en una actividad, a otra 
considerada vecina. Este caso se produce especialmente en las situaciones no construidas por el analista y toca de lleno a la validez ecológica. Si las relaciones del contexto con la comunicación pueden haber sido menos estudiadas directamente, el hecho es que "el contexto es una componente esencial de la comunicación y una fuente importante de la simplicidad y eficiencia" (Brown y Duguid 1994, p. 3). Las palabras “ contexto " y " comunicación ", ambas polisémicas, deberían estar siempre bien definidas y estudiadas de manera conjunta. Las investigaciones muestran que si el contexto actúa como un medio y un modulador del sentido de las comunicaciones, también puede haber efectos parásitos sobre las mismas. En todo caso, las investigaciones sugieren que comunicación y contexto deben estudiarse conjuntamente, sobre todo para definir más adecuadamente la actividad efectiva y su concepción.

\section{La simulación}

Los problemas que se plantean por las relaciones entre el laboratorio y el campo son muy similares a los encontrados por las investigaciones sobre la simulación como herramienta de formación. Estos problemas se han vuelto cada vez más evidentes debido a la creciente complejidad de los sistemas técnicos y a su impacto en la complejidad de los empleos y la capacitación correspondiente. La revisión de las relaciones entre la situación de laboratorio y la situación de campo no puede dejar de mencionar el examen de las relaciones entre la situación a simular y su simulación : se trata de los mismos tipos de problemas que se presentan en ambos casos. Consideremos aquí la formación : la cuestión fundamental que se le plantea al formador que desea utilizar la simulación es la de diseñar un simulador que facilite el aprendizaje de la tarea que será luego ejecutada. Para esto, es evidente que no es suficiente con construir un simulador que imite simplemente en apariencia la tarea a aprender. Esta fidelidad facial no carece de interés, pero resulta totalmente insuficiente cuando se trata de una tarea compleja. El simulador debe permitir al aprendiz el adquirir la competencia deseada y esto sólo se puede lograr con un simulador que lo prepare para la adquisición de los mecanismos de la actividad, incluso si a veces se trata de un simulador que no se parece facialmente a la tarea simulada. La literatura sobre estos temas es frondosa y de calidad diversa : en tal sentido, solo podemos ser alusivos sobre este punto.

Un trabajo colectivo de Bainbridge y Ruiz Quintanilla (1989) tiene toda una parte destinada a la habilidad y a la simulación como herramienta de formación : presenta detalladamente los encuadres analíticos, una metodología y diversos ejemplos extraídos del sector industrial. Hace también hincapié en la importancia de un análisis previo de la actividad, para poder desarrollar una simulación con fines formativos. Más recientemente, Fauqet-Alekhine y Pehuet (2011) han presentado un libro dedicado, también, a "la mejora de las prácticas profesionales a través de la simulación". Dicho libro habiendo sido diseñado con la colaboración directa de especialistas que siguieron el diseño y el funcionamiento en el terreno del dispositivo por ellos propuesto.

Por último, cabe destacar el trabajo de Pastré (2011), citado anteriormente, que incluye un capítulo titulado "dispositivos de aprendizaje construidos a partir de situaciones" en el cual se proponen métodos de análisis que permiten planificar el diseño de simuladores. Ofrece varios ejemplos y comentarios en los cuales tuvo participación directa (p. 269) como, por ejemplo, un simulador de resolución de los problemas del ajuste de una 
prensa a inyección en la industria del plástico y un dispositivo de simulación de la poda de la vid (p. 273). El comentario de estos casos es muy instructivo y constituye una buena introducción metodológica que puede interesar a los formadores.

\section{Conclusión}

21 El tema del ciclo de estudio en el cual se inscribe la síntesis presentada por Reuchlin es sumamente revelador en su propia formulación de las preocupaciones de los psicólogos en el momento en el cual fue escrito. En ese período aún quedaban rastros de los intensos debates de 1968, acerca del alcance práctico de las investigaciones de laboratorio, así como la significación de los resultados de dichas investigaciones, tanto para las personas, como para la sociedad. Los debates desarrollados acerca de las relaciones entre las investigaciones desarrolladas en ambos lados, estaban más centrados en las relaciones y los objetos de dichas investigaciones, que en el lugar de ejecución en el cual debería ponerse el énfasis. Estas discusiones harían emerger un cierto número de distinciones que van a mantenerse vivas generando el interés y el debate entre los psicólogos de las diferentes especialidades. Las expresiones tales como "la psicología aplicada" como la de "acoplamiento o dialéctica laboratorio / campo" no deben ser tomadas literalmente, sino más bien como la designación de un contenido que debe ser siempre explicitado. Reuchlin ha realizado una importante contribución a lo que se podría describir, de manera más general, como las relaciones entre la teoría y la práctica, pero aún queda mucho por hacer para la psicología en este campo, sin olvidar la finalidad de la investigación y sus relaciones con el contexto y con otras disciplinas que podrían resultar involucradas.

\section{BIBLIOGRAFÍA}

Bainbridge, L., \& Ruiz Quintanilla S.A. (Eds.) (1989). Developing skills with information technology, Chichester, UK: Wiley.

Brown, J.S. \& Duguid, P. (1994). Borderlines issues: social and material aspects of design. HumanComputer Interaction, 9, 3-36.

Fauquet-Alekhine, P. \& Pehuet, N. (2011). Améliorer les pratiques professionnelles par la simulation. Toulouse : Octares. Version anglaise : Simulation Training : Fundamentals and Applications. Springer International Publishing AG Switzerland.

Guérin, F., Laville, A., Dianellou, F., Duraffourg, J. \& Kerguelen, A. (1997). Comprendre le travail pour le transformer : la pratique de l'ergonomie. Montrouge: ANACT.

Hollnagel, E. (1993). Human reliability analysis. Context and control. London : Academic Press.

Lahy, J.-M. (1932). Les fondements scientifiques de la psychotechnique. L'hygiène Mentale, 27, 10, 273-302. (Un extrait de cet article figure dans un numéro spécial sur le centenaire de la société de psychologie française de la revue Psychologie française, Mars 2000, 45, 1, 273-277).

Laboreal, Volume $12 \mathrm{~N}^{\circ} 1$ | 2016 
Leplat, J. (1982). Le terrain, stimulant (ou obstacle) au développement de la psychologie cognitive. Cahiers de psychologie cognitive, 2, 2, 115-130.

Leplat, J. (2001). La gestion des communications par le contexte, Perspectives interdisciplinaires sur le travail et la santé. Pistes, 3-1.

Leplat, J. (2006). Les contextes en formation. Éducation permanente, 166, 1, 29-48.

Ombredane, A. \& Faverge, J.-M. (1955). L'analyse du travail. Paris : PUF.

Pastré, P. (2011). La didactique professionnelle. Approche anthropologique du développement chez les adultes. Formation et pratiques professionnelles. Paris : PUF.

Reuchlin, M. (1973). La psychologie appliquée a-t-elle des méthodes propres ? Dans M. Reuchlin (s/d). Traité de psychologie appliquée, tome 2. Méthodes (p. 5-18). Paris : PUF.

Reuchlin, M . (1977). Psychologie. Paris : PUF. ( $4^{\circ}$ éd.).

Wallon, H. (1930/1946). Principes de psychologie appliquée. Paris : A. Colin.

\section{AUTORES}

\section{JACQUES LEPLAT}

Groupe de Recherche et d'Etude sur l'Histoire du Travail et de L'Orientation (GRESHTO)

Centre de Recherche sur le Travail et le Développement (CRTD)

Conservatoire National des Arts et Métiers (CNAM)

41, Rue Gay Lussac 75005 Paris

France

jacques.leplat@wanadoo.fr 